\title{
Guest Editorial: Special Issue on Analytics for Local Events and News
}

\author{
Amr Magdy ${ }^{1} \cdot$ Xun Zhou $^{2} \cdot$ Daniel B. Neill ${ }^{3}$
}

Published online: 23 April 2020

(C) Springer Science+Business Media, LLC, part of Springer Nature 2020

This special issue contributes to better understanding of the impacts of the global urbanization process and new data sources on different aspects of society, lives, and activities at a localized scope. The issue papers study opportunities, challenges and cutting-edge techniques for local events and news analytics. This topic has gained considerable attention due to the advances in software and hardware technologies, together with the process of rapid urbanization globally over the last decade. These advances have changed the ways people interact as groups, both offline (physically), and online (virtually).

The growth and diversity of the urban population have led to more frequent events of different types ranging from sports games and traffic congestion to ad-hoc gatherings and social protests. Such events create multiple and substantial impacts on public safety, traffic, health, and business. In addition, online forums and social media have emerged as a new generator and information source for events and news. In 2016, social media outstripped television as a news source for young people for the first time in history. Nevertheless, both online and offline events and news play important roles in modern societies. Consequently, identifying, forecasting, and understanding events and news has emerged as an important topic. By nature, events and news have spatial and temporal extents, suggesting that they are localized social phenomena. In fact, they have generated several challenges and research problems that are inherently spatio-temporal problems. Addressing these problems at a local scale is both interesting and challenging due to the various sources of data, e.g., social media,

Amr Magdy

amr@cs.ucr.edu

Xun Zhou

xun-zhou@uiowa.edu

Daniel B. Neill

daniel.neill@nyu.edu

1 Department of Computer Science and Engineering, University of California, Riverside, Roverside, CA 92521, USA

2 Tippie College of Business, University of Iowa, Iowa City, IA, USA

3 Center for Urban Science and Progress, New York University, Brooklyn, NY 11201, USA 
traffic sensors, vehicle trajectories, and location-based check-ins, that help to address the topic. This variety in data sources brings several research challenges including dealing with large volumes of data, high levels of heterogeneity, and noisy user-generated data.

This special issue includes the following six papers:

[1] "Techniques for Efficient Detection of Rapid Weather Changes and Analysis of their Impacts on a Highway Network", by Adil Alim, Aparna Joshi, Feng Chen, and Catherine T. Lawson.

[2] "Local Trend Discovery on Real-time Microblogs with Uncertain Locations in Tight Memory Environments", by Abdulaziz Almaslukh, Amr Magdy, Ahmed M Aly, Mohamed F Mokbel, Sameh Elnikety, Yuxiong He, Suman Nath, and Walid Aref;

[3] "Crosstown Traffic - Supervised Prediction of Event Impact on Urban Traffic", by Nicolas Tempelmeier, Stefan Dietze, and Elena Demidova.

[4] "Building Socially-Enabled Event-Enriched Maps", by Faizan Ur Rehman, Imad Afyouni, Ahmed Lbath, Sohaib Khan, and Saleh Basalamah.

[5] "Enhancing Local Live Tweet Stream to Detect News", by Hong Wei, Jagan Sankaranarayanan, and Hanan Samet.

[6] “Online Influenza Epidemiological Modeling by Social Media Assimilation”, by Liang Zhao, Jiangzhuo Chen, Feng Chen, Fang Jin, Wei Wang, Chang-Tien Lu, and Naren Ramakrishnan.

The six papers provide a variety of thoughtful ideas and techniques that serve multiple purposes. Papers [1], [3], and [6] studies prominent types of events, weather changes, traffic events, and epidemic diseases, respectively. Meanwhile, papers [2], [4], and [5] provide generic techniques to handle a wide-variety of events or news. From another perspective, papers [1], [3], [4], and [6] propose event-related techniques, paper [5] proposes news-related techniques, while paper [2] provides fundamental aggregation techniques that could be used for discovering both news and events. Collectively, the six papers cover different perspectives for analyzing the rich available datasets for localized news and events.

We thank all the authors and reviewers for their valuable contributions and commitment to producing this special issue timely, as well as Springer editors and staff for their support.

Publisher's Note Springer Nature remains neutral with regard to jurisdictional claims in published maps and institutional affiliations. 